\title{
Pinocchio migrant et postcolonial : parcours de subjectivation entre Europe centrale, Italie, et Afrique \\ - Chiara Mengozzi
}

Sommaire : L'article porte sur deux récentes adaptations des Aventures de Pinocchio du célèbre écrivain italien Carlo Collodi : le roman Occhio a Pinocchio (2006) de Jarmila Očkayová, auteure italophone d'origine slovaque, et la pièce Pinocchio nero (2005), dirigée par Marco Baliani et interprétée par un groupe d'enfants des rues de Nairobi. Nous nous proposons de démontrer que ces deux auteurs, malgré les différences indéniables entre leurs œuvres (lieu de production et de réception, destinataire et genre littéraire), partagent la même stratégie postcoloniale à l'égard du "texte source " : ils s'approprient une œuvre du canon littéraire occidental en la transformant en une allégorie des procès de subjectivation/assujettissement des marginaux, qu'il s'agisse des (écrivains) immigrés en Italie ou des garçons de rue des bidonvilles kenyans. Notre approche des adaptations souhaite valoriser l'effet rétroactif que celles-ci peuvent avoir sur le texte de départ, notamment la capacité de dégager ou mettre en relief des significations négligées ou restées en marge des lectures précédentes du texte adapté. Nous aboutirons ainsi à une nouvelle interprétation du chef-d'œuvre de Collodi.

Mots-clés: Pinocchio ; adaptation ; subjectivation ; postcolonial ; Baliani, Marco ; Očkayová, Jarmila

\section{Introduction}

Soutenant avec Linda Hutcheon que le critère de " fidélité » emprunté à la morale ne devrait pas guider l'analyse des adaptations dans la mesure où celles-ci sont " secondes", mais en aucun cas "secondaires » (XIII), nous porterons notre attention sur deux des adaptations dont a fait l'objet l'œuvre pour la jeunesse la plus connue, traduite et adaptée 
au monde, Le avventure di Pinocchio de Carlo Collodi (alias Carlo Lorenzini), publiée d'abord dans la revue II giornale per i bambini, puis, en format livre, en $1883 .{ }^{1}$ La première adaptation ne prévoit pas un changement de code vis-à-vis du texte source : il s'agit du roman Occhio a Pinocchio, écrit en italien par Jarmila Očkayová, auteure d'origine slovaque vivant en Italie depuis 1974, et publié en 2006. La seconde, Pinocchio nero, une transcodification du récit au théâtre, a été dirigée par Marco Baliani, dramaturge italien, acteur et initiateur du courant du théâtre-récit, ${ }^{2}$ qui a porté sur scène en 2005 une remarquable version de Pinocchio, au terme d'un long travail avec un groupe d'enfants des rues de Nairobi au Kenya.

Deux questions se posent d'entrée de jeu, I'une concernant le corpus, et l'autre, d'ordre méthodologique. Pourquoi choisir de comparer, parmi les innombrables adaptations de Pinocchio, des versions italo-(tchéco)slovaque et italo-kényane? Que pourrait justifier un rapprochement aussi inusité ? Malgré les probables réserves qu'une critique postcoloniale " orthodoxe » pourrait avancer étant donnée la provenance des auteurs, l'opération d'Očkayová et de Baliani peut pleinement être qualifiée de postcoloniale, $d^{\prime}$ 'une part, parce que la création d'une catégorie de textes sur la base du critère autobiographique est fort contestable (celuici ne constituant nulle garantie, ni de qualité ni de résistance), et d'autre part, parce que les deux auteurs adoptent une stratégie fondamentale de la littérature postcoloniale : ils s'approprient une œuvre du canon littéraire occidental et s'en servent comme instrument d'agentivité. Le roman écrit par Očkayová et la pièce de théâtre conçue et dirigée par Baliani, bien qu'éloignés I'un de l'autre pour maintes raisons (lieu de production et de réception, genre littéraire, destinataire ${ }^{3}$ ) empruntent à Collodi l'histoire des aventures d'un enfant-marionnette dans I'Italie post-unitaire et, grâce à une dialectique entre permanence et innovation, la transforment en une allégorie des procès de subjectivation/assujettissement des marginaux, qu'il s'agisse des (écrivains) immigrés en Italie ou des garçons des rues des bidonvilles kényans. Certes, comme nous le démontrerons par I'analyse des traits formels des adaptations choisies, la valeur des deux œuvres en question ne se réduit pas à leur portée politique. Néanmoins, celle-ci doit être mise en premier plan, d'autant plus qu'entre la fin du XIXe siècle et le début du XXe, Pinocchio a été plusieurs fois remanié, réécrit et prolongé à des fins de propagande en accord avec les idéologies et les projets pédagogiques de l'Italie libérale, puis fasciste. Le roman, qui a connu lors de sa publication un succès immédiat et éclatant bien au-delà des attentes de son auteur, a rapidement inspiré de nombreux autres textes, parmi lesquels méritent d'être rappelées, pour I'influence qu'elles ont eue sur les jeunes lecteurs, les Pinocchiate signées par des auteurs sympathisants 
du régime fasciste dans une visée anticommuniste ou pour célébrer le colonialisme italien en Afrique et ridiculiser les autochtones. ${ }^{4}$ Quelques décennies plus tard et après un long détour à travers l'Europe centrale (en passant par la Russie) et le Kenya, le chef-d'œuvre de Collodi retourne à son pays d'origine sous une forme qui rappelle le «talking back postcolonial » (Ashcroft et al.), pour que les lecteurs italiens puissent I'apprécier de nouveau et y voir inscrit non seulement un parcours de normalisation progressive des marginaux, des rebelles comme Pinocchio, mais aussi un message d'émancipation sociale et culturelle pour les subalternes.

Le corpus à présent justifié, reste à préciser I'approche. Dans quel ordre faut-il étudier les adaptations par rapport au texte adapté ? S'il est vrai que faire l'expérience d'un texte en tant qu'adaptation signifie ne pas ignorer le dialogue intertextuel avec son texte d'origine, rien ne nous oblige à procéder dans la lecture, et dans I'analyse, en respectant l'antériorité chronologique du texte adapté. Au contraire, comme le suggère Linda Hutcheon dans son livre incontournable, A Theory of Adaptation, le fait par exemple de regarder le film inspiré d'un roman avant de (re)lire ce dernier pourrait nous aider à réviser nos préjugés et surtout à mettre définitivement hors jeu des questions telles que

"Cette adaptation est-elle fidèle à l'original ? ",

"Est-elle plus ou moins réussie que sa source?", et ce, en faveur de questions beaucoup plus pertinentes et bien synthétisées dans la règle d'or du journalisme : qui a adapté quoi, quand, où, pourquoi et comment? Au lieu de commencer par Collodi pour évaluer ensuite les écarts par rapport au texte source, nous partirons des adaptations pour revenir à Collodi dans un deuxième temps. Cette démarche nous permettra de mesurer l'effet « rétroactif » que I'adaptation peut avoir sur le texte de départ et donc de dégager des significations insoupçonnées ou restées en marge des interprétations précédentes du célèbre roman italien.

\section{Et maintenant, à moi la parole !}

Je m'appelle Pinocchio et je veux vous raconter mon histoire. De mon point de vue. Oh, j'imagine déjà ce que vous êtes en train de penser et je crois qu'il vaut mieux apporter immédiatement une précision : dans mon cas, il ne s'agit ni d'un cas d'antonomase, ni d'une fourberie d'épigone pour attirer l'attention avec la célébrité d'autrui. Je voudrais que cela soit clair : je suis Pinocchio. Ce Pinocchio-là, oui ! (Očkayová, Occhio a Pinocchio 7$)^{5}$

C'est ainsi, avec cette prise de parole résolue que s'ouvre le roman de Jarmila Očkayová. ${ }^{6}$ Après cent vingt-cing ans de récits associés à son personnage et sédimentés dans l'imaginaire des lecteurs, Pinocchio 
s'approprie son histoire de marionnette sans fils, à I'identité incertaine entre l'humain et le végétal : "L'aspect humain et l'âme millénaire de la forêt. Voilà ce qu'est une vraie marionnette » (12). ${ }^{7}$ Pinocchio, désirant devenir interprète et conteur des légendes millénaires de la forêt d'où il provient, nous raconte son long apprentissage à travers de nombreuses et douloureuses épreuves. Une fois sorti du tronc par I'acte de création de Geppetto, Pinocchio sera jugé et rejeté par les autres maîtres-artisans, même s'il n'a commis d'autre crime que celui d'être inclassable, à la fois naturel et artificiel, non plus arbre et pas encore humain, créature de la forêt et être fabriqué par les hommes, ni tout à fait étranger, ni complètement assimilé au sein de la communauté humaine :

- C'est Pinocchio, précisa Geppetto.

- C'est un orphelin, précisa Maître Sapin.

- C'est un balourd, précisa Maître Cerise.

- C'est un intrus, précisa Maître Peuplier.

- Un bouc émissaire, précisa Maître Platane.

- Un être embryonnaire à l'origine inconnue, précisa Maître Cyprès.

- Une œuvre du diable, précisa Maître Châtaignier.

- Un mystère à dévoiler, précisa Maître Saule.

- Une occasion à rattraper au vol, précisa Maître

Noyer. $(23)^{8}$
Les mésaventures de Pinocchio sont innombrables : accablé par le chagrin, séparé de Geppetto qui semble I'avoir oublié, suivi par le terrible marionnettiste Mangiafoco (qui ne peut pas lui pardonner d'avoir incité les autres marionnettes à la rébellion et voudrait le rattacher à des fils pour limiter sa liberté d'action), déçu par ses semblables (les autres marionnettes soumises à Mangiafoco), entortillé par le Chat et le Renard, Pinocchio finit par être enchaîné et enfermé dans une cabane-prison-requin sans possibilité d'en sortir. ${ }^{9}$ Cependant, c'est précisément cette série d'injustices et de cruautés infligées à Pinocchio qui pousse le lecteur à s'interroger sur la valeur et le sens des dichotomies qui guident le comportement des Maîtres, par exemple celle entre l'humain et l'inhumain. Si les bourreaux de Pinocchio peuvent l'emprisonner et le maltraiter sans aucun sentiment de culpabilité, c'est parce que, à leurs yeux, Pinocchio étant étranger, différent, " métisse », ne peut pas faire partie à plein titre de la communauté humaine ( « tant qu'on tient ligotée une marionnette en bois, on peut donner aux gens mille justifications à cet égard, et cela sans aucun effort particulier ; mais si c'est un homme qui se trouve enchaîné, la question change » [186]). ${ }^{10} C^{\prime}$ est leur attitude, toutefois, tellement féroce, barbare et aveugle (c'est-à-dire incapable de reconnaître chez « autrui » à la fois l'autre et le semblable), qui finit par se montrer " inhumaine », privée de toute empathie.

Le séjour de Pinocchio auprès de la communauté des humains se solde par un échec : il est à la fois 


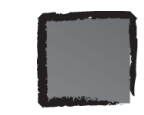

\section{Une fois sorti du tronc par l'acte de création de \\ Geppetto, Pinocchio sera jugé et rejeté par les autres maîtres-artisans, même s'il n'a commis d'autre crime que celui d'être inclassable....}

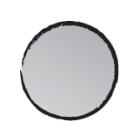

exposé aux regards méfiants des passants et à la merci des Maîtres qui le contrôlent et le criminalisent en utilisant le langage comme un instrument de domination des plus faibles. ${ }^{11}$ Le retour de Pinocchio à la forêt, quant à lui, semble à jamais impossible, non seulement à cause de sa captivité mais aussi parce que, après son long exil dans la ville, il se croit désormais dans l'incapacité de comprendre la langue de la forêt.

Si la conclusion paraît désespérée, il convient de bien soupeser les derniers mots du roman, qui permettent d'en tempérer le côté tragique : lorsque Pinocchio semble être sur le point de mourir (" les muscles des bras tremblaient de faiblesse et les yeux brûlaient sous l'effet de la chaleur et le cœur battait irrégulièrement à cause de l'excès de fatigue et de solitude . . . . Ses forces l'abandonnèrent ensuite et il s'écroula », 190), ${ }^{12}$ un narrateur externe intervenant dans l'épilogue, le seul passage du roman où Pinocchio ne parle pas à la première personne, conclut de la façon suivante : «Il s'accrocha à la seule chose qui lui restait : son imagination. Du cœur de la forêt, de son centre même, le grand faucon s'envola et visa droit vers le plateau de la cabane-requin » (190). ${ }^{13}$ Empêché de sortir de sa cabane-prisonrequin (allégorie des camps de réfugiés et des centres de rétention administrative éparpillés sur le territoire de la «forteresse Europe » et de ses zones frontalières), ${ }^{14}$ Pinocchio réagit à I'hostilité et aux mensonges des autres personnages au moyen du rêve, de la créativité et de l'imagination. Ceux-ci lui permettent d'abattre les murs matériels et symboliques élevés par les Maîtres dans le but de niveler les diversités, d'exclure ou de renfermer tout individu étranger, déviant, anomal, non conforme à la majorité. Chez Collodi, les mensonges du protagoniste ont un caractère foncièrement ambivalent : d'un côté, ils manifestent une forte puissance autodestructrice ; de l'autre, ils 
révèlent l'irréfrénable et indomptable vitalité créative du personnage. Očkayová, elle, préfère exalter seulement ce deuxième aspect et évoquer le pouvoir politique qui peut découler d'un usage particulier de la langue, de I'imagination et de la fiction littéraire.

Le roman d'Očkayová paraît se renfermer sur lui-même, car cette référence à l'imagination suggère que Pinocchio peut enfin réaliser son rêve et devenir conteur, s'opposant ainsi à l'oubli et à l'anonymat auxquels son histoire et celle d'autres étrangers, immigrés, réfugiés, exilés, subalternes comme lui semblent être condamnées dans la communauté des Maîtres-artisans, c'est-à-dire dans la société d'arrivée. Autrement dit, le roman que l'on vient de lire (re)commence lorsqu'il s'achève : Pinocchio est désormais capable de raconter les histoires de la forêt, puisque il vient de raconter la sienne, à partir de son point de vue. II meurt marionnette pour renaître narrateur, ${ }^{15}$ dorénavant conscient et fier de sa nature indécidable, de sa double appartenance (non plus végétal, pas encore humain ; à la fois arbre et enfant), car c'est précisément son identité de frontière qui lui donne le privilège du double regard, qui lui permet de traduire les histoires de l'ailleurs (« en le traduisant dans votre langue » est un syntagme fréquent dans le roman) ${ }^{16}$ et ainsi d'entamer une communication avec les lecteurs. Si dans la diégèse il n'y a pas d'harmonie possible entre Pinocchio et sa nouvelle communauté (les mots de Pinocchio sont toujours détournés et incompris), une complicité est au contraire envisageable avec les lecteurs dans l'acte littéraire dont il est protagoniste en tant que narrateur, comme en attestent les nombreux appels aux lecteurs qui sont évoqués dès la première phrase (« Je veux vous raconter mon histoire ») et par le traitement ludique de la langue. Pinocchio, dans son parcours d'apprenti conteur, profite de sa condition marginale pour redécouvrir la langue italienne, capter des sens nouveaux, inventer des étymologies extravagantes et funambulesques : " J'étais plus que désespéré : j'étais exaspéré. Ce qui revient à dire que j'étais désespéré triple, s'il est vrai que le préfixe "dé" indique le numéro deux et le préfixe "exa" en revanche le six. Ce qui reste en ôtant les préfixes, paradoxalement, c'est l'espoir » (129). ${ }^{17}$

Pour comprendre pourquoi I'histoire de Pinocchio est devenue, sous la plume d'Očkayová, le récit d'un personnage suspendu entre deux mondes et visant, au moyen de la traduction, à conquérir une parole qui ne soit pas hétérodirigée, mais libre et autonome, il est fondamental de contextualiser cette adaptation : qui est I'auteure? Quand et où I'adaptation a-t-elle été écrite et publiée ? Pourquoi l'auteure a-t-elle choisi de réécrire Pinocchio?

Jarmila Očkayová a émigré dans les années 1970 de la Slovaquie (à l'époque Tchécoslovaquie) vers I'Italie, où elle a continué son activité d'écrivaine en liant son nom à ce que I'on appelle couramment « littérature de la migration », terme qui désigne l'ensemble des textes 
littéraires écrits en italien par les immigrés (Mengozzi, Narrazioni ; Quaquarelli). Les premiers exemples de cette production apparue en Italie au début des années 1990 sont des romans-témoignages prévoyant une collaboration entre un auteur immigré, censé fournir le contenu, l'expérience «brute», et un journaliste ou écrivain italien, censé prêter son soutien linguistique pour mettre l'expérience en récit (Bouchane et al. ; Khouma et Pivetta ; Methnani et Fortunato). Si Očkayová insiste sur l'acte d'appropriation de la parole (du narrateur hétérodiégétique chez Collodi, on passe à un narrateur autodiégétique) et sur la narration de l'histoire du point de vue de Pinocchio (focalisation interne), c'est parce que l'auctorialité n'est pas une question anodine dans la littérature de la migration. La parabole dessinée par Pinocchio dans ce roman (du discours nié, exproprié, détourné, à la possibilité d'un discours autonome) peut être donc lue comme une allégorisation de l'histoire de la littérature migrante : si, dans un premier temps, les représentants de cette production avaient comme but principal celui de bousculer, parfois à l'aide des collaborateurs italiens, les stéréotypes sur les immigrés mis en circulation par les médias, les protagonistes de cette littérature se sont ensuite progressivement émancipés de la présence du collaborateur/garant italien qui, tout en rendant possible la publication, avait aussi encadré, contrôlé, normalisé et autorisé les textes des immigrés pour les rendre conformes à I'horizon d'attente des lecteurs italiens. ${ }^{18}$ Chez Collodi, Pinocchio est déjà un personnage caractérisé par la course et la fugue. ${ }^{19}$ Dans I'adaptation de Očkayová, non seulement essaie-t-il tout au long du récit de s'échapper des rôles et des définitions identitaires imposés par les autres personnages (les Maîtres-artisans et Mangiafoco en particulier), mais il finit par s'éloigner de ses deux "pères » en prenant congé à la fois de Geppetto (qui fait seulement une brève apparition au départ) et de Collodi dont on peut enfin se débarrasser, puisque Pinocchio a trouvé le courage de raconter son histoire sans intermédiaires.

Afin de comprendre ce qui a conduit Očkayová à faire de l'auctorialité le cœur idéal de son adaptation, nous devons examiner le " où » et surtout le «quand » de l'adaptation. Publié en 2006, donc plus de quinze ans après la naissance d'une littérature migrante en italien, ce roman ne correspond plus à la phase de I' « urgence de la voix », mais plutôt à celle de la recherche d'une citoyenneté parmi les écrivains italiens. Pour une écrivaine étrangère telle Očkayová, le fait de réécrire un classique de la littérature italienne, de se situer donc dans I'héritage de la tradition tout en rivalisant avec celle-ci, signifie trouver une stratégie d'affranchissement du ghetto de la littérature migrante en faveur de la littérature italienne contemporaine tout court. Mais pourquoi adapter précisément Le avventure di Pinocchio? 
Si Očkayová a toujours démontré de l'intérêt pour la littérature de jeunesse (elle a publié en 1998 un roman intitulé Appuntamento nel bosco, destiné aux enfants), il convient de rappeler la position que le roman de Collodi occupe à l'intérieur du canon littéraire italien. Bien que n'étant pas un Bildungsroman au sens strict du terme (puisque la structure cyclique et répétitive du livre aurait pu proliférer à l'infini et la transformation finale du personnage ne semble pas être le résultat d'un parcours de développement et rédemption linéaires et progressifs), ${ }^{20}$ les aventures/mésaventures de Pinocchio se prêtent à incarner les conflits sociaux, politiques, et culturels auxquels les Italiens, en tant qu'individus et en tant que nation, faisaient face à un moment crucial de leur histoire-celui qui suivait I'unification nationale, (presque) accomplie sur le plan politique, mais encore inachevée sur le plan social, culturel et linguistique.

Bien que le sens du livre et I'intention de l'auteur restent ambivalents et indécidables (est-ce que c'est

la transgression qui prévaut ou plutôt le nécessaire retour à l'ordre, l'assujettissement à la morale dominante ?) et que les représentants du pouvoir soient souvent ridiculisés, le roman de Collodi traite, de manière plus ou moins ironique, tous les grands thèmes qui intéressaient de près la construction de la jeune Italie libérale : I'éthique du travail, I'instruction, le respect de l'autorité, l'émancipation de la pauvreté, la construction des liens sociaux (Poettinger). Ce n'est pas un hasard, en effet, si Suzanne Stewart-Steinberg
(2007) a choisi Pinocchio comme métaphore directrice de son livre consacré à la construction de la nation italienne entre I'unification politique et l'instauration de la dictature : tout comme I'enjeu principal pour I'Italie de l'époque semble être précisément celui de trouver son identité en se libérant des influences des États étrangers pour ainsi entreprendre son propre chemin vers la modernité, le personnage de Pinocchio cherche la sienne en oscillant constamment entre la soumission à l'ordre constitué et la recherche de son autonomie.

$S^{\prime}$ il est vrai que derrière les aventures/mésaventures $\mathrm{d}^{\prime}$ une marionnette-enfant qui s'apprête à devenir adulte ${ }^{21}$ se cachent également les difficultés et les contradictions qu'implique le processus politique, social et culturel d'unification nationale, le fait de s'approprier ce récit signifie pour Očkayová de contribuer implicitement à imaginer l'avenir de la nation italienne. Un avenir que déjà Collodi avait préféré laisser ouvert en passant sous silence le nom du pays que Pinocchio et son père rejoignent, vers la fin de leurs tribulations, sur le dos $d^{\prime}$ un thon après être demeurés dans le ventre du requin. Nonobstant la présence du chat et du renard (que Pinocchio abandonne à leur destin), du grillon-qui-parle, de Lucignolo mourant et de la jolie fillette/chèvre/Fée aux cheveux (toison) bleu nuit, Pinocchio et Geppetto ne sont probablement pas retournés à leur pays/village/maison d'origine : déposés sur le rivage par le thon, ils ne se dirigent pas vers leur première habitation mais se mettent à chercher un nouveau logement. 
Puisqu'il est une figure charnière entre l'« ici » (il détient l'aspect et la langue de la communauté humaine) et I'« ailleurs » (son origine est végétale et il reste en connexion avec le langage de la forêt), le Pinocchio d'Očkayová introduit la pluralité dans la nation. Autrement dit, il suggère que la cohésion culturelle et linguistique impliquée dans le concept même de nation devrait céder le pas à l'hétérogénéité, que la nation italienne devrait être prête non seulement à valoriser les diversités régionales ou linguistiques qui la caractérisent depuis sa naissance, mais aussi à offrir I'hospitalité aux différences introduites par les récents mouvements migratoires transnationaux. En résumé, le Pinocchio

\section{... il suggère que la} cohésion culturelle et linguistique impliquée dans le concept même de nation devrait céder le pas à l'hétérogénéité ....

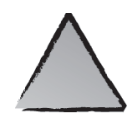

d'Očkayová demande implicitement aux lecteurs, lectrices si fréquemment interpellés dans son récit d'imaginer une conduite distincte de celle des Maîtres-artisans qui l'ont rejeté en tant qu' « autre » et d'assurer au contraire une place à différentes façons d'être Italien, Italienne.

Que le roman d'Očkayová suggère cette idée plurielle d'identité culturelle et nationale est confirmé par les choix formels, en particulier celui de réécrire le texte de Collodi (lui aussi le résultat d'un mélange de traditions hétéroclites, notamment entre le récit toscan et le conte de fées français dont Collodi était traducteur) ${ }^{22}$ à travers deux autres médiations fusionnées et incorporées dans le texte de départ. L'hypothèse que nous proposons est que les transformations opérées sur le roman de Collodi dérivent en partie du fait d'avoir entrelacé au texte italien les contes populaires du folklore slovaque recueillis par Pavol Dobšinský au XIX̀̀me siècle et magistralement traduits en italien par Očkayová elle-même en 1988 (d'où, par exemple, l'importance accordée à la forêt dans sa version de Pinocchio) et la très célèbre adaptation russe des Aventures de Pinocchio signée par Alexis Tolstoï et publiée en 1936. ${ }^{23}$ À cette dernière, Očkayová emprunte probablement les trois éléments suivants : la place centrale accordée à Mangiafoco, que Tolstoï renomme Karabas Barabas ; 
le thème de la rébellion que Pinocchio voudrait guider contre ce dernier pour permettre aux marionnettes de se débarrasser de leur maître diabolique (il s'agit de l'intrigue principale de la version russe) ; et, enfin, un détail structurel—à l'instar du récit de Tolstoï, le roman d'Očkayová suit l'enchaînement des épisodes collodiens jusqu'à l'apparition de Mangiafoco pour s'en détacher ensuite presque complètement.

En conclusion, non seulement Očkayová métaphorise, à travers la figure de Pinocchio, la condition d'immigré à laquelle, à tort ou à raison, ${ }^{24}$ elle s'identifie, mais elle fait aussi en sorte que son adaptation dépasse les seuils de la fiction littéraire. Si son personnage qui voudrait être accueilli dans la communauté d'arrivée tout en restant attaché à ses origines ne semble accomplir son désir qu'au moment où il prend la parole à la première personne pour raconter son histoire aux lecteurs, I'auteure elle aussi, à travers la réécriture d'un classique—qui prévoit, comme toute adaptation, la variation dans la permanence$s^{\prime}$ inscrit à plein titre dans la tradition littéraire italienne tout en gardant son bagage culturel antérieur à la migration depuis la Tchécoslovaquie vers I'Italie. En rendant le texte de Collodi à la fois plus dépaysant (pour les lecteurs italiens, ainsi obligés de relire Le avventure di Pinocchio à partir d'un autre point de vue) et plus familier (pour elle, grâce à l'emploi de thèmes et motifs de provenance slave), Očkayová trouve et impose sa propre voix dans l'establishment littéraire italien.

\section{"Le chemin vers soi passe à travers le monde et les autres ${ }^{25}$}

C'est précisément pour transformer la vie et pour permettre aux garçons des bidonvilles kényans de trouver un statut d'existence que Marco Baliani et ses collaborateurs ont accepté de partir en Afrique dans le cadre du projet Children in Need financé par I'association non gouvernementale Amref, dans le but de monter un laboratoire théâtral avec une vingtaine de garçons «Chokora » (" poubelle » en Swahili) provenant du quartier Dagoretti, le plus défavorisé de Nairobi. Le pari initial était de tirer ces garçons de la rue et des décharges où ils vivaient sans famille, privés de tous moyens et étourdis par la colle. Dans le beau livre qui narre la genèse du spectacle, Baliani les décrit ainsi :

Si on les appelle Chokora, c'est parce que ces garçons ne sont pas des personnes, ce sont des choses, ils n'ont pas de statut d'existence, ils ne possèdent pas d'identité, ce sont des non-personnes qui survivent aux marges de ce qui est déjà marginal. L'unique possibilité de reconnaissance est celle d'appartenir à une bande, à un groupe, à la « Base ». Pour le reste du monde, ils n'existent pas, ils peuvent être tués, violés, battus ou bien jetés en prison quand la police montre ses muscles et veut décrasser les rues du centre-ville. Je comprends que mon travail théâtral doit commencer ici, par la question du manque de dignité et d'identité individuelle. $(21)^{26}$ 
Au fil des jours, l'enjeu du laboratoire se transforme et le pari devient plutôt celui d'entraîner ces garçons afin de leur permettre de monter sur scène dans un vrai spectacle. Le choix de Pinocchio surgit par hasard. Un soir, à la fin de la première semaine du laboratoire, Baliani commence à raconter Le avventure di Pinocchio au moyen de geste et d'un anglais titubant que les garçons peuvent comprendre tant bien que mal (le traducteur-médiateur arrivera seulement plus tard). Le lendemain, l'un des garçons-Chokora, imite sous le regard amusé des autres les mouvements d'un corpsmarionnette. Pour Baliani, « Si dans un seul après-midi ils ont réussi à s'approprier le corps d'un personnage, cela veut dire que cette matière les touche $\gg(53),{ }^{27}$ c'est que l'histoire de Pinocchio racontée par Collodi résonne avec leur vie : il est pauvre, affamé, sans origine, analphabète, asservi, vendu, blessé, et même pendu à un arbre dans l'épisode le plus inquiétant où s'achevait la première version du roman publié par Collodi en 1881. À partir de ce moment, la figure de Pinocchio deviendra le fil conducteur des exercices théâtraux proposés par Baliani aux jeunes garçons. Le spectacle, qui sera présenté en 2004, d'abord en Afrique dans plusieurs théâtres de Nairobi et dans le quartier Dagoretti d'où les garçons proviennent puis ensuite à Rome et à Palerme devant un public de 900 personnes, est seulement le couronnement d'un parcours de formation de deux ans. À l'entraînement physique et technique, pendant lequel Baliani insiste sur les mouvements du corps-marionnette et apprend aux garçons les rudiments du métier d'acteur, s'ajoutent progressivement des exercices qui permettent aux garçons de se raconter les uns aux autres en employant le personnage fictif de Pinocchio comme un masque, une forme de protection. L'un des exercices, par exemple, consiste à utiliser l'épisode collodien où Pinocchio se brûle les pieds sur le brasero comme prétexte pour mettre en commun les histoires cachées derrière les cicatrices qui marquent leurs corps.

Leur pièce, Pinocchio nero, "n'est pas tant la mise en scène d'un roman ", écrit Franco Nasi, " mais plutôt la mise en vie d'un texte : un texte qui se fait expérience vécue, un texte qui se transforme et qui, en se transformant, transforme la vie » (211). ${ }^{28} \mathrm{La}$ singularité de la destinée de ces garçons s'inscrit en effet dans le roman collodien en le chargeant de sens nouveaux, tandis que la mise en scène de ce récit leur donne une identité et modifie leur statut social. Le spectacle (et ses revenus) leur a permis de transformer leur indigence absolue en une misère plus digne, leur a donné un véritable passeport leur permettant de prendre un avion, les a réintégrés dans ce qui restait de leur famille, ou a créé de nouveaux liens au sein de la communauté de Dagoretti. De plus, il les a persuadés que chacun d'entre eux est une personne avec une histoire singulière digne d'être racontée et transfigurée sur scène. Peu importe que les lieux et les dates de naissance inscrits dans leurs documents soient pour la plupart inventés, puisque c'est précisément grâce à 
la fiction qu'ils ont pu conquérir une voix et un statut d'existence. C'est parce qu'ils ont joué un rôle et qu'ils ont fait l'expérience de s'identifier à un personnage romanesque qu'ils ont pu finalement découvrir qu'ils sont quelqu'un.

Que le vécu et le théâtre s'entrelacent et s'influencent réciproquement, voilà ce qui est suggéré à tout moment dans le spectacle, et en particulier dans les scènes d'ouverture et de clôture, deux véritables seuils qui établissent et confondent à la fois l'espace de la vie et celui de la fiction. Le spectacle s'ouvre sur le chant a cappella d'un garçon qui assume le rôle du narrateur, souvent présent sur scène :

Ne nous appelez pas Chokora

$C^{\prime}$ est un nom horrible

Il fait mal au cœur

Frères, aimons-nous I'un I'autre

Les problèmes nous ont poussés à nous enfuir

En nous obligeant à vivre dans la rue, à mendier un peu d'argent et un repas

Les problèmes nous ont chassés de nos écoles

En nous obligeant à vivre dans la rue ${ }^{29}$

Dans cette scène d'ouverture, l'acteur interpelle directement le public tout en évoquant la collectivité qui se cache derrière I'histoire de cette marionnette nommée Pinocchio. De même que la chanson d'ouverture, quoique chantée par un seul acteur, établit aussitôt la dimension chorale de ce spectacle, I'incipit archiconnu de Collodi (« Il était une fois . . . Un roi ! Non, il était une fois . . . un morceau de bois »), ${ }^{30}$ ici prononcé par le narrateur en italien, prévoit l'entrée de tous les garçons du groupe qui se dispersent sur scène, rigides comme des troncs d'arbres. Parmi ces garçons-troncs aux mouvements tendus et parfaitement coordonnés entre eux, le narrateur en choisira un, lui donnera la vie selon un rite masaï, et un nom, Geppetto. Le menuiser sculptera ensuite sa marionnette dans l'espoir de pouvoir en profiter pour gagner tant bien que mal sa vie avec des spectacles ambulants.

Entre la première scène où, comme on vient de le voir, la vie vire progressivement vers la fiction, et la dernière où, comme on le verra par la suite, la fiction et la vie se superposent, se succèdent seize scènes qui suivent de près l'agencement et le contenu du texte collodien, y compris les épisodes les plus violents, comme celui de la pendaison de Pinocchio. Les seules exceptions sont des contractions imposées par la durée du spectacle et quelques remarquables adaptations culturelles qui renvoient à la fois aux traditions animistes masaï et au contexte concret de la vie précaire des jeunes acteurs. C'est un Masaï qui vend à Geppetto en échange de son vieux manteau I'abécédaire de Pinocchio dans un décor à la fois très simple et capable d'évoquer tout de suite l'atmosphère d'un marché africain typique. Ce n'est pas Pinocchio qui se brûle les pieds par inattention et inexpérience : 


\author{
$\bigcirc$ \\ Pinocchio pourrait être \\ vu ... comme un résidu \\ de la modernization....
}

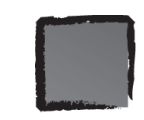

trois autres garçons de la rue sont responsables de cet acte effectué par pure méchanceté, pendant qu'il dormait. Une fois au Pays des Jouets, c'est le rêve d'avoir des chaussures de soccer qui se réalise, dans une scène irrésistible où, au rythme d'une musique envoûtante choisie par les garçons eux-mêmes, les acteurs dansent tous ensemble et jouent avec un ballon imaginaire. Le personnage de la Fée, d'après Baliani le plus difficile à concevoir dans la mesure où il s'agit d'un concept intraduisible en kiswahili (« les garçons ont plus facilement accepté l'idée d'un morceau de bois parlant que celle d'une femme magique aux cheveux bleus qu'ils ne savent pas comment et où situer dans leur expérience $»^{31}$ écrit-il), ne correspond pas à une femme aux cheveux bleus, mais plutôt à une présence vague, impalpable, à un esprit de la nature au visage caché derrière un masque africain blanc et introduite par une musique éthérée, ni rassurante ni tout à fait inquiétante.

Dans la conclusion du spectacle, la dimension collective toujours sous-jacente à l'adaptation fait de nouveau irruption : Pinocchio est désormais responsable de lui-même et de son papa. Quand il sort du ventre du requin, tous les Pinocchio avancent en portant sur leur dos, chacun à sa façon, Geppetto, le père perdu et enfin retrouvé. Après avoir appuyé la silhouette de la marionnette non pas sur une chaise, comme dans la version de Collodi, mais sur une vieille boîte à ordures en fer-blanc (un objet qui renvoie manifestement au contexte des bidonvilles), I'un des Pinocchio prend la parole : «Quel drôle d'air j'avais quand j'étais marionnette! Et comme je suis content d'être un garçon! ${ }^{32}$ Le roman de Collodi s'arrête ici, mais le spectacle continue : les différents Pinocchio se lancent dans une dernière danse accompagnée par un célèbre morceau de jazz, Her Cab de Gianluigi Trovesi e Gianni Coscia. Il s'agit d'une danse clownesque et véritablement libératoire, à la différence des épisodes précédents, où 
la danse est seulement le prélude à une métamorphose rabaissant la marionnette à un état inférieur, comme lors de la transformation en âne au Pays des Jouets. Tandis que la musique décélère peu à peu, les mouvements des acteurs ralentissent jusqu'à ce qu'ils s'écroulent par terre. Les Pinocchio tombent raides morts au sol pour renaître garçons. Un par un, les acteurs se lèvent, sortent leur passeport et, le montrant fièrement, crient joyeusement leur noms : «Je m'appelle Alex Wachaga et maintenant j'ai un passeport! Je m'appelle Joseph Muthoka, je m'appelle Wilson Franko, je m'appelle George Kamau etc. ».33

Lorsque les garçons brandissent ainsi leurs documents, nous ne sommes tout à coup plus au théâtre, mais le passage entre la fiction et la vie réelle a eu lieu sans que le rideau ne se baisse. En effet, il n'y pas de discontinuité palpable entre l'une et l'autre, non seulement parce que les jeunes acteurs, grâce à la médiation de Baliani et ses collaborateurs, se sont appropriés I'histoire de Pinocchio en la transformant en une métaphore de leur condition, mais aussi parce que c'est grâce à l'entraînement et la préparation pour la mise en scène qu'ils ont pu conquérir un statut d'existence, concrètement, avec une identité reconnue par l'État, et symboliquement, avec une identité narrative correspondant à la capacité acquise de se raconter et de partager ces récits entre eux, avec les membres de la communauté de Dagoretti et avec un public plus large, jusqu'en Italie.
Cependant certaines questions restent encore sans réponse : "Pinocchio, » se demande Baliani, " qu'a-t-il gagné et qu'a-t-il perdu au cours de cette transformation? » (146). ${ }^{34}$ En effet, à y regarder de plus près, cette question en cache trois distinctes : Pinocchio, en tant que roman de Collodi, en tant que personnage métaphorisant la condition de ces garçons et en tant que garçon Chokora acteur de ce spectacle, qu'a-t-il perdu et gagné ? Autrement dit, cette adaptation a-t-elle fait " violence » au texte de Collodi en supprimant, condensant ou transformant des scènes, ou bien l'a-t-elle enrichi en lui octroyant des sens nouveaux et imprévisibles, peut-être déjà en puissance dans la version dite originale ? Et encore, Baliani et ses collaborateurs ont-ils fait "violence » à ces garçons en traduisant/métaphorisant leur expérience au moyen d'un récit familier aux lecteurs occidentaux et en ayant le dernier mot dans le livre Pinocchio nero, qui présente aux lecteurs italiens le but du programme financé par Amref et les phases principales de la mise en scène? Enfin, que veut dire concrètement et qu'implique la transformation de la vie de ces garçons permise par le projet théâtral ?

Baliani ne propose ni développe les trois différentes pistes de réflexion implicites dans la citation tirée de son livre. II se limite à nous dire qu'à son avis les garçons ont payé un prix pour les changements qu'ils on vécu (ils ont abandonné la rue et les drogues et parfois retrouvé leur famille) : ils ont dû, par exemple, 
se soumettre à des règles et à un entraînement fatigant, ils ont dû écouter leur metteur en scène et ses collaborateurs qui leur dictaient ce qu'ils devaient faire et comment ils devaient se comporter. En deux mots, ils ont perdu cette liberté apparente dont ils étaient jaloux après des années passées dans la rue. Mais, en réalité, précise encore Baliani, de même que dans le récit de Collodi la liberté initiale de Pinocchio est seulement illusoire puisque, en étant seul, pauvre, affamé et analphabète, il passe d'un esclavage à l'autre, de même la liberté de ne pas se soumettre à la moindre règle dans leur vie comportait une non-existence juridique, un destin fait de misère, violences, et abus. Tout compte fait, c'est aussi un avantage pour eux d'avoir perdu leur trompeuse liberté initiale pour gagner d'autres libertés, notamment celle de voyager ou de se séparer temporairement ou définitivement de leur pays. Autrement dit, leur histoire nous démontre qu'entre autonomie et dépendance, entre subjectivation et assujettissement, il y a toujours un rapport de co-implication.

Par ailleurs, I'adaptation de Baliani, au cours de la migration du texte depuis I'Italie vers le Kenya, nous permet de revenir au roman de Collodi et de le relire avec un regard nouveau pour valoriser ce qui reste en marge ou caché dans la plupart des interprétations. En particulier, elle nous permet, entre autres, de mettre en relief la double nature indécidable du texte collodien (à la fois histoire de normalisation disciplinaire et d'autodétermination). Les aventures/mésaventures de Pinocchio ne nous racontent pas seulement I'histoire d'une infraction réitérée aux normes imposées, mais aussi I'histoire de la difficile recherche d'une identité, de la libération de l'esclavage matériel imposé par la misère.

Le personnage du roman collodien est non seulement un marginal, mais également un déchet, un matériel de rebut (Belpoliti), un bout de bois que Maître Cerise voudrait destiner à la cheminée et que Geppetto, dans sa pauvreté absolue (il n'a ni repas digne de ce nom, ni moyen de se réchauffer : sa cheminée est peinte sur le mur), cherche à transformer en marionnette pour en tirer un petit revenu. Pinocchio pourrait être vu, plus précisément, comme un résidu de la modernisation, celle qui a investi I'Italie lors de I'unification nationale et que connaissent actuellement plusieurs pays africains où un capitalisme déréglé entraîne le dépérissement des campagnes et de I'économie locale, I'urbanisation forcée, I'accroissement des périphéries-bidonvilles et la dissolution des liens sociaux et familiaux. Collodi a choisi comme protagoniste de son roman un enfant né par hasard dans un milieu défavorisé et souffrant constamment de faim (le mot « faim » apparaît une quarantaine de foi dans le roman). Pinocchio passe d'un esclavage à l'autre non seulement parce qu'il est paresseux et irrévérencieux mais aussi et surtout parce qu'il n'a aucun moyen de se défendre des maîtres et des adultes qui profitent de lui. ${ }^{35}$ Pinocchio est presque brûlé par Mangiafoco bien décidé 
à rôtir sa viande sur le feu, il est obligé d'assumer le rôle de chien de garde d'un poulailler. Transformé en âne, il doit d'abord se laisser humilier dans un cirque ; puis il risque de se faire écorcher par des hommes qui voulaient faire de sa peau un tambour ; il devient presque le repas d'un pêcheur désireux de le faire frire avec d'autres poissons ; et même Geppetto, son père, au tout début du récit, voulait lui aussi I'exploiter dans un théâtre de marionnettes ambulant.

Les critiques littéraires d'Italie et d'Europe, désormais « adultes » et opulentes, ont souvent regretté le caractère normatif de la conclusion du roman, en accordant leur préférence aux infractions et incartades de la marionnette plutôt qu'à l'image finale du jeune garçon aux yeux bleus, travaillant pour subvenir aux besoins de son vieux père (Lavagetto ; Tempesti). Audelà des préférences personnelles pour l'un ou l'autre aspect du roman collodien, la plupart des critiques ont tendance à voir dans les derniers deux chapitres (XXXV et XXXVI) une conclusion brusque et presque postiche, qui s'accorde mal avec l'esprit dominant du roman :

During a careful reading of the novel [. . .] one can't really help but to feel the difference in concept and tone between this last chapter and the previous body of the story, and it comes natural to suspect that Collodi might have consciously failed to connect it to the rest of the novel. [. . .] the purely moral content was so alien to his creative sensibility that the result came out awkward and markedly lacking in inspiration. (Arnaudo 384)

Outre le fait que cette interprétation de la conclusion se présente comme une spéculation abstraite ${ }^{36}$ et qu'elle ne prend en considération ni la continuité entre les quinze premiers chapitres et les vingt-etun suivants, ${ }^{37}$ ni les « jeux de miroir » entre les deux conclusions (celles des chapitres XV et XXXVI), ${ }^{38} \mathrm{ni}$ le caractère moralisateur propre aussi à la première version du roman, ${ }^{39}$ elle est limitée en ce qu'elle schématise l'histoire de Collodi et en simplifie le contenu. Autrement dit, si la célèbre adaptation de Disney banalise indiscutablement le roman collodien en accentuant excessivement le côté consolatoire et moralisateur, de la même manière on peut juger come réductrice toute interprétation qui voit dans le roman une suite d'aventures et de transgressions brusquement tronquées par une conclusion postiche et non convaincante où le personnage (contre la " vraie intention » de l'auteur) comprendrait soudainement l'importance du travail, de l'instruction, de l'autorité parentale, etc. À notre avis, il ne s'agit pas de mettre en valeur la force anti-autoritaire de la première version du roman (jusqu'au chapitre XV) au détriment de la deuxième (en particulier de la conclusion) et de son caractère normalisateur, mais plutôt de comprendre que le roman met en scène, tout au long de son développement, une dynamique 
complexe entre liberté et influence en montrant I'interdépendance foncière qui existe entre contrôle et autonomie, normes et transgressions, émancipation, et asservissement : la liberté initiale de la marionnette et son refus de l'autorité s'avèrent en réalité une forme atroce d'esclavage à plusieurs maîtres, tandis que la soumission finale aux normes représente paradoxalement la condition pour devenir un « vrai garçon », c'est-à-dire un sujet libre et autonome.

Le spectacle des garçons kényans nous permet de mettre en lumière et d'apprécier l'ambivalence du roman, et en particulier de sa conclusion, d'y voir non seulement un décevant retour à l'ordre mais aussi un important projet d'émancipation sociale et culturelle pour tous ceux qui sont relégués dans la marginalité. La transformation de Pinocchio en garçon, qui marque l'acquisition d'une identité et d'une conscience de soi, est accompagnée de la métamorphose du contexte dans lequel il vit, ce qui indique un réel et considérable changement de classe sociale :

La pièce aux murs nus de la cabane en paille était devenue une jolie chambre meublée et décorée avec une élégante simplicité. Sautant du lit, il découvrit aussi un costume neuf, un nouveau chapeau et une paire de bottines en cuir qui lui allèrent parfaitement. [. . .] [I]I trouva un petit porte-monnaie d'ivoire [. . .] Mais les quarante sous n'étaient plus de vulgaires pièces en cuivre. Le porte-monnaie contenait quarante sequins en or, flambant neuf et brillant de tous leurs feux. (Collodi, Les aventures 199) $)^{40}$

Pour un lecteur adulte et riche cela est peut-être ennuyant; pour les enfants qui lisent Pinocchio depuis plus d'un siècle, c'est tout simplement magique ; du point de vue des subalternes $c^{\prime}$ est un projet à accomplir.

Quant aux normes auxquelles Pinocchio semble se soumettre à la fin du récit, il faudrait encore rappeler qu'il n'y pas de coïncidence parfaite entre les règles que Geppetto, la Fée-aux-cheveux bleus, et le Grillon-qui-parle voudraient lui imposer et le mode de vie assumé par Pinocchio. Il est vrai que Pinocchio accepte à la fin du récit de Collodi de tourner la noria du matin au soir comme un âne : un travail très peu édifiant qui causera la mort de son ami La Mèche/ Lucignolo (lequel n'a jamais pu abandonner l'aspect animal) et dont Pinocchio prendra la place. Mais, pour Pinocchio, devenu un enfant sage et responsable, tourner la noria au service de Giangio le maraîcher est un véritable choix de vie, grâce auquel il peut procurer du lait à son vieux père et mettre de côté un joli magot qui lui permettra de changer les conditions matérielles de son existence. Il est vrai, en outre, que la dernière étape de I'humanisation de Pinocchio advient au moment où le protagoniste comprend finalement l'importance de la lecture et de l'écriture, mais au lieu d'aller à l'école où les livres tuent (on fait ici référence 
à l'épisode formidable où les compagnes de classe lancent grammaires, abécédaires, et toutes sortes de manuels contre Pinocchio, provoquant finalement un traumatisme crânien au pauvre Eugène avec " un traité d'arithmétique qui pesait des tonnes », 120), ${ }^{41}$ Pinocchio sort de sa condition d'analphabète en apprenant seul à lire à partir d'un livre " auquel il manquait les premières et les dernières pages » $(196)^{42}$ et à écrire sur un cahier avec une «brindille en guise de plume » qu'il « trempait dans un petit récipient rempli de jus de mûres et de cerises » (196). ${ }^{43}$ Autrement dit, sa métamorphose est d'un côté un acte d'assujettissement aux normes sociales que Pinocchio avaient refusé tout au long du récit, et de l'autre, un acte d'autodétermination. La pièce Pinocchio nero rend explicite et accentue l'importance de ce thème déjà présent dans les pages collodiennes, par exemple en diminuant le rôle accordé à la Fée qui n'apparaît qu'une fois et qui, en guise de réponse à Pinocchio qui lui demande de le transformer en un vrai garçon, dit : " Je ne peux pas te transformer. Ton destin dépend seulement de toi $»{ }^{44}$ accentuant ainsi la possibilité dont dispose le personnage de décider de son avenir à la première personne.

Certes, la pièce n'a pas entièrement constitué un acte d'autodétermination de la part des garçons, ne serait-ce que parce qu'elle s'inscrit dans un projet humanitaire dont le côté paternaliste ne peut pas être facilement contourné. Malgré le fait que chaque scène ait été le résultat d'une longue négociation entre les jeunes acteurs et le dramaturge italien, ils n'en reste pas moins que les rapports de pouvoir entre les sujets impliqués ne peuvent être facilement passés sous silence ou renversés. Certes, c'est Baliani et ses collaborateurs qui ont guidé les garçons dans un parcours d'émancipation sociale et culturelle en leur donnant un cadre d'intelligibilité et un nom et un visage connus (celui de Pinocchio) qui permettent aux spectateurs occidentaux de comprendre la condition des protagonistes. Néanmoins, tout parcours de subjectivation est simultanément un parcours d'assujettissement; il va de pair avec l'acceptation des normes qui d'un côté nous surveillent et de l'autre nous donnent un statut d'existence aux yeux des autres et de I'État : « neither submission nor mastery, » affirme Judith Butler dans The Psychic Life of Power, " is performed by a subject; the lived simultaneity of submission as mastery, and mastery as submission, is the condition of possibility for the emergence of the subject » (117).

Collodi, qui a choisi pour son roman une conclusion ambivalente montrant à la fois l'évolution positive du personnage qui découle de sa soumission aux normes (il se réveille jeune garçon aisé) et le versant dramatique de cet encadrement (la marionnette est appuyée à une chaise, sans vie, les bras ballants), démontre qu'il en était parfaitement conscient; Baliani et ses acteurs savaient également que l'assujettissement aux normes sociales et théâtrales était le prix à payer pour être enfin reconnus en tant que personnes; pour acquérir, en interprétant le 
rôle d'un personnage romanesque, un statut d'existence face à la communauté d'appartenance. Après tout, selon une étymologie accréditée, le mot « personne » désignait antiquement le masque utilisé par les acteurs. Aucune surprise, alors, si la différence entre la « personne » et le " personnage » risque d'être presque imperceptible.

\section{Notes}

${ }^{1}$ La bibliographie relative aux réécritures, traductions et adaptations de Pinocchio est désormais pléthorique. Cf., parmi les travaux récents les plus intéressants, Beckett ; Dedola et Casari ; Pezzini et Fabbri ; Scrivano ; et Wunderlich et Morrissey.

${ }^{2}$ Le théâtre-récit ou théâtre de narration est l'un des plus importants courants de la dramaturgie italienne contemporaine. Parmi ses représentants figurent Baliani, Paolini, Curini, et Celestini. Il s'agit d'un théâtre civil qui puise dans la tradition incarnée par Dario Fo. La figure centrale est le narrateur qui raconte-souvent seul sur scène et muni d'un nombre restreint d'accessoires—des épisodes méconnus et gênants de l'histoire italienne récente. Voir, par exemple, Guccini.

${ }^{3}$ La pièce s'adresse à un public d'adultes et d'enfants (kényans et italiens). Le roman s'adresse principalement aux adultes.

${ }^{4}$ Luciano Curreri a recueilli quatre exemples de Pinocchiate fascistes publiées entre 1923 et 1944 dans lesquelles le pantin brutalise les communistes et tourne en ridicule le Négus d'Éthiopie. Il faut ajouter que la moquerie envers les autochtones n'est pas une prérogative des Pinocchiate publiées sous le régime fasciste: certains textes écrits plus tôt partagent le même esprit, sans s'avérer pour autant ouvertement colonialistes. Voir par exemple le roman d'Eugenio Cherubini, Pinocchio in Africa, publié en 1903, dans lequel Pinocchio finit par être couronné roi de tous les Africains, qui sont par ailleurs représentés de manière grotesque. D'autres classiques destinés aux enfants ont servi à de semblables fins didactico-politiques, tels Le petit chaperon rouge (voir Lodolini, Cappuccetto rosso nell'Africa Orientale, 1936) et Tintin (en particulier Tintin au Congo, 1931), qui a été interdit aux États-Unis (mais non en Belgique) pour son contenu discriminatoire. Sur la littérature de jeunesse italienne à l'époque coloniale, voir les remarquables essais de Colin publiés en 2003 et en 2012.

5 « Mi chiamo Pinocchio e voglio raccontarvi la mia storia. Raccontarvela dal mio punto di vista. Oh, immagino ciò che state pensando e credo convenga fare subito una precisazione : il mio non è un caso di antonomasia, né tantomeno di furbizia da epigono che vuole attirare l'attenzione con altrui celebrità. Vorrei che fosse chiaro : io sono Pinocchio. Quel Pinocchio, sì » (7). Sauf mention contraire, toutes les traductions sont de l'auteure. Les pages des citations, par conséquent, font référence à la version italienne.

${ }^{6}$ Si Očkayová et ses romans sont très connus des chercheurs en littérature de la migration, la bibliographie relative à ce roman est, d'une façon surprenante, plutôt pauvre. Voir Camilotti et Barbarulli. 
${ }^{7}$ « Le sembianze umane e l'anima millenaria del bosco, ecco che $\cos ^{\prime}$ è un vero burattino » (12).

8 « - Questo è Pinocchio - precisò Geppetto. - Questo è un orfano - precisò il maestro Abete. - Uno sbandato - precisò il maestro Ciliegia. - Un intruso - precisò il maestro Pioppo. - Un capro espiatorio - precisò il maestro Platano. - Un essere embrionale di origine sconosciuta - precisò il maestro Cipresso. - Un'opera del diavolo - precisò il maestro Castagno. - Un mistero da svelare - precisò il maestro Salice. - Un'occasione da cogliere al volo precisò il maestro Noce » (23).

${ }^{9}$ Ici Očkayová réunit deux épisodes différents du roman de Collodi : le chapitre XXI, où Pinocchio est asservi par un paysan qui lui met un épais collier clouté et l'oblige à faire le chien de garde près d'un poulailler, et le chapitre XXXIV, où le protagoniste est avalé par un redoutable requin " grand comme une montagne ». Selon un procédé récurrent dans le roman, le Pinocchio d'Očkayová dénonce et dévoile la métaphorisation de sa vie faite par Collodi en nous racontant ne pas avoir été avalé par un requin, mais avoir plutôt été emprisonné dans une baraque.

10 « fino a che tieni legato un burattino di legno, alla gente puoi dare mille giustificazioni, in proposito, e senza nemmeno tanti sforzi ; ma se a essere in catene è un uomo la faccenda cambia » (186).

11 Dans l'adaptation d'Očkayová, Pinocchio ne ment pas. Ce choix, à première vue surprenant, permet en effet d'accentuer un aspect qui dans le texte de Collodi risque de passer au second plan. Dans son étude sur le mensonge en littérature, Lavagetto ( $L a$ cicatrice di Montaigne, pp. 159-63) affirme que Pinocchio a acquis de façon imméritée la réputation de menteur, voulant souligner par là que l'allongement de son nez a fini par obscurcir l'autre rapport fondamental entre le personnage et le mensonge, à savoir le fait d'être sans cesse victime des mensonges des autres, auxquels il croit aveuglément par naïveté, par inexpérience ou encore-peuton soupçonner-par goût de la provocation ou pour des intérêts personnels.

12 « i muscoli delle braccia gli tremavano dalla debolezza e gli occhi bruciavano dalla calura e il cuore perdeva colpi per troppa stanchezza e troppa solitudine [. . .] poi gli mancarono le forze e si accasciò » (190)

13 « Si aggrappò all'unica cosa che gli restava : la sua immaginazione. Dal cuore del bosco, proprio dal suo punto centrale, si levò in volo il grande falco e puntò dritto verso il pianoro del casotto pescecane » (190).

14 Il convient de rappeler ici la thèse de Giorgio Agamben, qui, dans Homo sacer, fait du « camp »le paradigme biopolitique de l'Occident. Ainsi, le Pinocchio d'Očkayová, réduit à sa pure existence biologique par le pouvoir (ici représenté par les Maîtres), se trouve renfermé dans un camp : un lieu d'inclusion/exclusion paradoxale de l'individu dans la sphère du droit, un lieu où se produit la politisation de la "vie nue ». À l'instar des Juifs dans I'Allemagne nazie, coupables du seul fait d'être Juifs, Pinocchio l'est du simple fait d'être un immigré.

15 Il s'agirait donc d'une conclusion à la fois tragique et heureuse, exactement comme celle écrite par Collodi. Emilio Garroni, dans sa brillante analyse qui reste à l'heure actuelle l'une des plus perspicaces du livre de Collodi, soutient que l'auteur, voulant dans un premier temps conclure l'œuvre au chapitre XV avec la pendaison du pantin, a finalement accompli, dans la version définitive, son désir de tuer le protagoniste, en dédoublant le 
personnage : une stratégie qui permet en effet d'intégrer la mort de Pinocchio tout en laissant vivre le garçon (lorsque Pinocchio se réveille garçon, il trouve le vieux pantin sans vie, appuyé contre une chaise).

16 « A tradurlo nel vostro linguaggio » (9).

17 " Ero più che disperato : ero esasperto. Il che è come essere disperato triplo, se è vero che il prefisso "di" indica il numero due e il prefisso "esa" invece il sei. Ciò che resta togliendo i prefissi, paradossalmente, è la speranza » (129).

18 Il faudrait toutefois remettre partiellement en question l'idée d'une progressive émancipation de la figure du collaborateur italien, qui semble être trop téléologique et simpliste. S'il est vrai que le nombre de romans et récits écrits sans la coopération d'un co-auteur italien augmente considérablement, il ne faut pas négliger que les écritures en collaboration connaissent un nouvel essor dans les années 2000 (voir à ce propos Mengozzi, "Archivio »). II n'en demeure pas moins que la représentation linéaire et téléologique de l'évolution de cette production, bien qu'en partie fausse, reste dominante encore aujourd'hui pour son caractère consolatoire.

19 Asor Rosa appelle Pinocchio une « créature cinétique » (41). Dès que Geppetto le crée, Pinocchio commence à courir et s'enfuit aussitôt de la maison. À partir de ce moment, le topos de la course, de la fugue et de la poursuite se répète sans cesse dans le texte.

${ }^{20}$ Sur les séquences réitérées du roman, voir la célèbre analyse structurelle de Gérard Genot, qui en indique neuf principales : dessein, obstacle, hésitation, manquement, punition, repentir, épreuve, salut, et récompense.
21 Selon Suzanne Stewart-Steinberg, la rhétorique qui guidait le projet de "faire les Italiens » n'était pas tellement celle de l'émancipation des adultes, mais visait plutôt l'éducation des enfants : "Even when the focus was on the literal education of children, as in the case of Maria Montessori's work, the metonymic referent was in fact a nation composed only of children. It was this aspect that answered to the idea that [. . . I Italy—and this particularly when compared to other modern nations - was in a state of perpetual infancy. The childlike Pinocchio as a cultural icon must, therefore, be understood in these terms » (3).

22 Collodi traduisit les contes de Charles Perrault, Marie-Catherine D'Aulnoy et Jeanne-Marie Leprince de Beaumont et publia en 1876 un recueil de ces contes traduits et remaniés, I racconti delle fate.

23 Златното ключе или приключенията на Буратино / Zolotoj ključik ili Priključenija Buratino. Dans les pays socialistes, et en particulier en Russie, I'adaptation de Tolstoï était plus célèbre que I'œuvre italienne. Le texte russe fut traduit en tchèque très promptement (Zlatý klíček, aneb, Buratinova dobrodružství, 1937) et en slovaque en 1950 (Zlatý klúčik alebo Buratinove príhody). Deux nouvelles traductions parurent en 1957 et 1975.

24 Očkayová appartient non seulement à une élite intellectuelle (étant déjà écrivain avant la migration), mais elle arrive aussi en Italie en 1974, à savoir quand le terme d'immigré n'est pas répandu dans la presse et dans le discours publique italien. Očkayová décide ensuite de se joindre au groupe des écrivains dits migrants, ce qui veut dire que l'étiquette n'a plus alors seulement un effet ghettoïsant mais aussi de (auto)promotion.

25 «Cesta k sobě vede přes cestu do světa a k druhým ». La citation est tirée de Patočka (45-46). 
26 «Se li chiamano Chokora è perché questi ragazzi non sono persone, sono cose, non hanno uno statuto d'esistenza, non posseggono un'identità, sono non-persone, che sopravvivono ai margini di quello che è già marginale. L'unico segno di riconoscimento è quello dell'appartenenza alla banda, al gruppo, alla "Base". Per il resto del mondo non esistono, possono essere uccisi, violentati, malmenati oppure rinchiusi in prigione quando la polizia mostra i muscoli e ripulisce le strade del centro. Intuisco che il mio lavoro teatrale deve partire da qua, dalla mancanza di dignità e di identità individuale » (21). La référence à Agamben est ici de nouveau pertinente. Ces garçons, en effet, rappellent la notion d'Homo sacer : selon le droit romain, l'Homo sacer était l'individu qui, étant banni de la communauté d'appartenance, pouvait être tué par n'importe qui (sans aucune conséquence pour l'assassin), mais ne pouvait pas être sacrifié (dans les rituels religieux). L'Homo sacer, loin d'exister seulement à l'époque de la Rome antique, serait, selon Agamben, la figure clef de la (bio)politique occidentale, car elle montre le seuil $\mathrm{d}^{\prime}$ inclusion/exclusion où se trouve un individu réduit à sa « vie nue ». Les garçons Chokora dont Baliani nous parle occupent ce même seuil : privés d'existence juridique, ils sont exposés à toute sorte de violence destinée à rester impunie.

27 « Se in un solo pomeriggio sono riusciti a incamerare il corpo di un personaggio vuol dire che quella materia narrativa li tocca » (53).

28 Exception faite d'un mémoire de maîtrise (Vinazzani), I'essai de Franco Nasi-publié dans sa monographie touchant aux parodies, traductions et réécritures-représente, pour autant que nous sachions, le seul article académique consacré à cette pièce injustement négligée par les chercheurs. Nasi compare la version Disney de Pinocchio et celle de Baliani en insistant, à raison, sur le caractère édulcoré et manichéen (entre le bien et le mal, entre les personnages absolument positifs et négatifs) de la première. L'adaptation de Baliani, quant à elle, respecte I'ambivalence de certains personnages (comme la fée) et refuse de supprimer les scènes sombres du texte-source (entre autres, la pendaison de la marionnette), car la violence, les injustices et les punitions toujours disproportionnées subies par Pinocchio tout au long du récit font allusion à la vie dans la rue des garçons de Nairobi.

29 Le prologue est chanté en kiswahili, la langue principale du spectacle, pourvu de sous-titres en italien. De temps en temps, les acteurs prononcent des mots ou des phrases en italien, les passages les plus célèbres du roman.

30 « C'era una volta . . . un re ! No, c'era una volta un pezzo di legno » (I'incipit du spectacle élimine, bien sûr, la référence aux petits lecteurs).

31 « I ragazzi hanno accettato con più facilità l'idea di un pezzo di legno parlante che non la presenza di questa donna magica con i capelli blu che non sanno come e dove collocare nella loro esperienza »(Baliani 89).

32 Cette phrase (la dernière du roman de Collodi) est prononcée en italien par l'acteur : «Com'ero buffo, quand'ero un burattino ! E come sono contento di essere un bambino ». La variation par rapport au texte de Collodi est minime : «E come ora sono contento di essere diventato un ragazzino perbene » (Collodi, Pinocchio 526). Les citations en italien sont tirées de l'édition critique.

33 " Mi chiamo Alex Wachaga e ora ho un passaporto ecc. ». Ces dernières phrases sont prononcées en italien. 
34 " Ma nella trasformazione cosa ha perso e cosa ha guadagnato Pinocchio?» (146).

35 Collodi était particulièrement sensible à cette question : «Qu'il soit dit à notre honte, l'esclave n'est pas un privilège exclusif de l'Amérique! Même l'Europe prodigieusement civilisée a ses esclaves [. . .] des individus si déchus de tout droit civil que leur condition épouvantable ne leur laisse rien à envier à un cultivateur de canne à sucre » ["Sia detto a nostra vergogna, lo schiavo non è un privilegio esclusivo dell'America! Anche la civilissima Europa ha i suoi schiavi [. . .] degli individui, così decaduti da ogni diritto civile, che per la loro durissima condizione non hanno nulla da invidiare un coltivatore di canne da zucchero ». Lorenzini/Collodi publie l'article dont la citation est tirée en 1858, dans la revue La lente en le signant ZZTZZ. Sur cet aspect, voir Poettinger.

${ }^{36} S^{\prime}$ il est vrai que Collodi, dans un premier temps, s'était arrêté au chapitre $X V$, il n'en reste pas moins que la version définitive du texte, jamais remaniée par l'auteur et à laquelle le lecteur doit volens nolens se confronter, est celle qui aboutit au chapitre XXXVI. Les jeunes lecteurs de Collodi et le directeur de la revue lui ont tout simplement demandé de donner une suite au roman. Ils ne lui ont pas imposé de le conclure de cette manière.

37 La preuve en est que l'analyse structurelle de Genot s'applique sans difficulté aux deux parties.

38 Le chapitres XV et XXXVI s'achèvent tous deux avec la mort de la marionnette. Si dans le premier cas la mort représente une forme extrême et sadique de punition contre la transgression initiale du personnage, dans le deuxième, c'est une sorte de récompense pour la prise de conscience du personnage.
39 Les conclusions tragiques et cruelles sont typiques de la moralité des contes populaires qui visaient à éduquer l'enfant au moyen de punitions exemplaires, d'épilogues angoissants et anxiogènes. À cet égard, les versions du Petit chaperon rouge précédentes à celle des Grimm (y compris celle de Perrault, auteur traduit par Collodi) où le chasseur n'intervenait pas, peuvent être rapprochées de la première conclusion de Pinocchio. Si la première partie du roman présente elle aussi un aspect moralisateur et édifiant indéniable, le chapitre XXXVI manifeste, en revanche, un caractère moqueur et irrévérent qui s'accorde bien avec l'esprit des autres chapitres : après s'être réveillé garçon, Pinocchio ridicule son ancienne identité. Voir à ce sujet Bonanni.

40 « invece delle solite pareti di paglia della capanna, vide una bella camerina ammobigliata e agghindata con una semplicità quasi elegante. Saltando giù dal letto, trovò preparato un vel vestiario nuovo, un berretto nuovo e un pajo di stivaletti di pelle, che gli tornavano una vera pittura. [. . .] Aperto il portafoglio, invece dei 40 soldi di rame, vi luccicavano quaranta zecchini d'oro, tutti nuovi di zecca » (Collodi, Le avventure di Pinocchio 525).

41 «Vi lascio immaginare se era peso di molto» (Collodi, Le avventure 461).

42 « Al quale mancavano il frontespizio e l'indice » (522).

43 " un fuscello temperato a uso penna » che « intingeva in una boccettina ripiena di sugo di more e di ciliege » (523).

44 "Siwezi kukubalidisha. Hiio inategemea na wewe». 


\section{Ouvrages cités}

Agamben, Giorgio. Homo sacer : Il potere sovrano e la nuda vita. Einaudi, 1995.

Arnaudo, Marco. "Against Chapter XXXVI : Sequels and Remakes of Collodi's Pinocchio in Italian Literature. » Forum Italicum, vol. 41, no. 2, 2007, pp. 382-402.

Ashcroft, Bill, et al., dirs. The Empire Writes Back: Theory and Practice in Postcolonial Literatures. Routledge, 1989.

Asor Rosa, Alberto. "Le avventure di Pinocchio : Storia di un burattino di Carlo Collodi. » Letteratura italiana einaudi : Le opere, dirigé par Alberto Asor Rosa, Einaudi, 1995, vol. 3, pp. 3-87.

Baliani, Marco. Pinocchio nero : Diario di un viaggio teatrale. Rizzoli, 2005.

Barbarulli, Clotilde. « Di fronte al banchetto delle parole del potere, sogna, pinocchio, sogna . . . s Scrittrici migranti : la lingua, il caos, una stella. Edizioni ETS, 2010, pp. 97-112.

Beckett, Sandra. «Le Pantin persistent et protéiforme : réécritures de Pinocchio. » Quaderni d'Italianistica, vol. 25, no. 1, 2004, pp. 43-67.

Belpoliti, Marco. "Carlo Collodi, le avventure di Pinocchio. » II romanzo, vol. 4. Temi, luoghi, eroi, dirigé par Franco Moretti, Einaudi, 2003, pp. 773-85.

Bonanni, Veronica. «Riscrivere la fine di Pinocchio : Tra parola e immagine. » Between, vol. 2, no. 4, 2012. doi: dx.doi. org/10.13125/2039-6597/683.

Bouchane, Mohammed, et al. Chiamatemi Alì. Leonardo, 1990.

Butler, Judith. The Psychic Life of Power: Theories in Subjection. Stanford UP, 1997.

Camilotti, Silvia. "Per una scrittura eccentrica : Occhio a Pinocchio di Jarmila Očkayová. » Mediazioni, vol. 6, 2009. www.mediazioni. sitlec.unibo.it/images/stories/PDF_folder/document-pdf/
dossierBusinaro2009/camillotti.pdf.

Carreri, Luciano, dir. Pinocchio in camicia nera : quattro pinocchiate fasciste. Nerosubianco, 2011.

Cherubini, Eugenio. Pinocchio in Africa. Bemporad, 1903.

Colin, Mariella. « L'Afrique pour l'enfance : aventures et colonialisme dans les livres pour l'enfance et la jeunesse de l'Italie libérale. » Afrique coloniale et postcoloniale dans la culture, la littérature et la société italiennes, dirigé par Mariella Colinet et Enzo Rosario Laforgia, PU de Caen, 2003, pp. 63-82.

---. « Des colonies à l'empire fasciste. La conquête de l'Afrique racontée aux enfants italiens. »Strenæ, vol. 3, 2012. doi: dx.doi. org/10.4000/strenae.322.

Collodi, Carlo. Le avventure di Pinocchio : Opere, dirigé par Daniela Marcheschi, Arnoldo Mondadori, 1995, pp. 361-526.

---. Les aventures de Pinocchio : Histoire d'une marionnette, traduit par Claude Sartirano, Édition de la Seine, 2006. Disponible sur Canopé : Académie de Strasbourg, www.crdp-strasbourg.fr/je_lis_ libre/livres/Collodi_Pinocchio.pdf.

Dedola, Rossana, et Mario Casari, dirs. Pinocchio in volo tra immagini e letterature. Bruno Mondadori, 2008.

Dobšinský, Pavol. Il re del tempo e altre fiabe slovacche, traduit par Jarmila Očkayová, Sellerio, 1988.

Garroni, Emilio. Pinocchio uno e bino. Laterza, 1975.

Genot, Gérard. Analyse structurelle de Pinocchio. Industria tipografica fiorentina, 1970.

Gnisci, Armando. La letteratura italiana della migrazione. Lilith, 1998.

Guccini, Gerardo. La bottega dei narratori. Storie, laboratori e metodi di : Marco Baliani, Ascanio Celestini, Laura Curino, Marco Paolini, Gabriele Vacis. Dino Audino, 2005. 
Hergé. Tintin au Congo. 1931. Casterman, 1970.

Hutcheon, Linda. A Theory of Adaptation. Routledge, 2006.

Khouma, Pap, et Oreste Pivetta. Io venditore di elefanti : una vita per forza tra Dakar, Parigi e Milano. Garzanti, 1990.

Lavagetto, Mario. La cicatrice di Montaigne. Einaudi, 1992.

---. Lavorare con piccoli indizi. Bollati Boringhieri, 2003.

Lodolini, Armando. Cappuccetto rosso nell'Africa orientale. Cappelli, 1936.

Lorenzini, Carlo. «Episodi del teatro della pergola. » La lente, 3 mars 1858. Reproduit dans: Collodi, Carlo. Cronache dall'Ottocento, dirigé par Daniela Marcheschi, Ets Editrice, 1990, pp. 28-29.

Mengozzi, Chiara. «Archivio e mercato del vissuto : Su alcune scritture collaborative degli anni Duemila. » Transkurturelle italophone Literatur / letteratura italofona transculturale, dirigé par Martha Kleinhans et Richard Schwaderer, Königshausen \& Neumann, 2013, pp. 7-55.

---. Narrazioni contese : Vent'anni di scritture italiane della migrazione. Carocci, 2013.

Methnani, Salah, et Mario Fortunato. Immigrato. Bompiani, 2006.

Nasi, Franco. «Pinocchio tra Hollywood e Nairobi. » Specchi comunicanti : Traduzioni, parodie, riscritture. Medusa, 2010, pp. 195-221.

Očkayová, Jarmila. Appuntamento nel bosco. EL, 1998.

---. Occhio a Pinocchio. Cosmo lannone, 2006.

Patočka, Jan. Tělo, společenství, jazyk, svět. Oikoymenh, 1995.

Pezzini, Isabella, et Paolo Fabbri, dirs. Le avventure di Pinocchio :

Tra un linguaggio e l'altro. Meltemi, 2002.

Pinocchio nero, réalisé par Marco Baliani, vidéo par Angelo Loy,
Giunti, 2005.

Poettinger, Monika. « Un burattino in libertà nell'italia di fine ottocento. » Carlo Lorenzini : Dal Risorgimento all'Unità, dirigé par Cosimo Ceccuti et Antonia Ida Fontana, Associazione Culturale Pinocchio, 2011, pp. 127-82.

Quaquarelli, Lucia. Narrazione e migrazione. Morellini, 2015.

Sayad, Abelmalek. L'Immigration ou les paradoxes de l'altérité, vol. 1, L'Illusion du provisoire. 1991. Raison d'agir, 2006.

Scrivano, Fabrizio, dir. Variazioni Pinocchio : 7 letture sulla riscrittura del mito. Morlacchi, 2010.

Stewart-Steinberg, Suzanne. The Pinocchio Effect : On Making Italians, 1860-1920. The $U$ of Chicago P, 2007.

Tempesti, Fernando. "Chi era il Collodi : Com'è fatto Pinocchio. » Pinocchio, par Carlo Collodi, Feltrinelli, 1972, pp. 7-49.

Tolstoï, Alexis. /l compagno pinocchio, traduit par Luigi Garzone, Stampa alternativa, 1981.

---. Zlatý klíček, aneb, buratinova dobrodružství, traduit par Petr Denk, Melantrich, 1937.

---. Zlatý klúčik čiže buratinove príhody, traduit par Vladimír Pežanský, Obroda, 1950.

---. Zolotoj ključik ili priključenija buratino, traduit par Eric Hartley. 1936. Hutchinson's Books for Young People, 1947.

Vinazzani, Christian. C'era una volta ... venti ragazzi di strada : Marco Baliani e Pinocchio nero. Mémoire de maîtrise, Università degli Studi di Modena e Reggio Emilia, 2006.

Wunderlich, Richard, et Thomas J. Morrissey. Pinocchio Goes Postmodern: Perils of a Puppet in the United States. Routledge, 2002. 
Chiara Mengozzi, docteure en Littérature Comparée, est chercheuse affiliée au département des Lettres de I'Université Charles de Prague et post-doctorante auprès du CEFRES de Prague. Elle enseigne aussi la littérature française à l'Université de Hradec Králové. En tant que professeure invitée dans le cadre du programme EuroPhilosophie, elle a enseigné à I'Université Hosei de Tokyo. Ses recherches portent notamment sur les littératures migrantes et postcoloniales et les intersections entre philosophie et littérature. Parmi ces publications récentes, la monographie Narrazioni contese : Vent'anni di scritture italiane della migrazione (Carocci 2013). 